\author{
Rita Jacobs \\ Patrick M. Honore \\ Herbert Spapen
}

\section{Look before leaping into combining extracorporeal techniques to improve oxygenation!}

Accepted: 6 September 2015

Published online: 22 September 2015

(C) Springer-Verlag Berlin Heidelberg and ESICM 2015

A response to these comments can be found at: doi:10.1007/s00134-015-4055-z.

Dear Editor,

We would like to comment on the recently published case study by Zhang et al. on incorporating a continuous renal replacement therapy (CRRT) circuit into a venoarterial extracorporeal membrane oxygenator (VA-ECMO) [1]. Their reported findings must be interpreted with great caution. First, the type of ECMO treatment used is questionable. As indicated by the authors, the patient presented with neurogenic pulmonary edema, which is a typical non-cardiogenic acute respiratory distress syndrome (ARDS). No data on cardiac function were provided. According to the Extracorporeal Life Support Organization guidelines, VAECMO should be applied exclusively in cardiac failure, whereas venovenous ECMO is first-line therapy for refractory or life-threatening hypoxemia in patients with preserved or drug-sustained cardiac function [2,3]. Venoarterial venous (VAV) ECMO is most appropriate to secure upper body oxygenation in case of severe myocardial dysfunction. Peripheral arterial cannulation for VAV-ECMO is feasible, yet a central approach better "synchronizes" perfusion to oxygenation needs by more closely regulating oxygenated blood return to the coronary and cerebral circulation $[2,3]$. Second, the high metabolic oxygen demands of ARDS patients can only be met by providing a high (i.e., between 3 and $7 \mathrm{~L} / \mathrm{min}$ ) ECMO blood flow rate. ECMO flow rates of $500-1500 \mathrm{~mL} / \mathrm{min}$ effectively remove $\mathrm{CO}_{2}$ from the circulation but are unsuited for oxygenation purposes. Thus, implementing a CRRT device at a "meagre" blood flow rate of $300 \mathrm{~mL} / \mathrm{min}$ into an ECMO circuit clearly is inadequate for improving oxygen supply [3, 4]. Third, embedding a CRRT device in series with an ECMO circuit may have significant drawbacks [5]. Optimal performance of CRRT requires convenient anticoagulation therapy. Recent guidelines recommend the use of regional citrate as first-choice anticoagulant.

A CRRT-ECMO combination inevitably will enhance citrate clearance. Eventual resulting premature clotting is harmful because it reduces filter and circuit life-span, predisposes to hemodynamic instability, and leads to treatment inefficacy (e.g., prolonged downtime) and unwarranted blood loss [5]. In addition, increased shear stress, physical properties of the bypass circuit, the roller pump, and the hemofilter enhance red blood cell damage and lysis [5]. A hemolysisinduced rise of plasma-free hemoglobin and subsequent hemoglobinuria adversely affect renal tubular function and may cause acute kidney injury or delay renal recovery $[2,5]$.

Compliance with ethical standards

Conflicts of interest The authors declare to have no competing interests.

\section{References}

1. Zhang Z, Chen K, Ni H, Xu X (2015) Incorporation of a hemofilter circuit into venoarterial extracorporeal membrane oxygenation: a novel approach to provide more oxygenation. Intensive Care Med 41:729-730. doi:

10.1007/s00134-015-3706-4

2. Shekar K, Mullany DV, Thomson B, Ziegenfuss M, Platts DG, Fraser JF (2014) Extracorporeal life support devices and strategies for management of acute cardiorespiratory failure in adult patients: a comprehensive review. Crit Care 18:219

3. Gattinoni L, Carlesso E, Langer T (2013) Clinical review: extracorporeal membrane oxygenation. Crit Care $15: 243$

4. Richard R, Argaud L, Blet A, Boulain T, Contentin L, Dechartres A, Dejode JM, Donetti L, Fartoukh M, Fletcher D, Kuteifan K, Lasocki S, Liet JM, Lukaszewicz AC, Mal H, Maury E, Osman D, Outin H, Richard JC, Schneider F, Tamion F (2014) Extracorporeal life support for patients with acute respiratory distress syndrome: report of a consensus conference. Ann Intensive Care 4:15

5. Jacobs R, Honoré PM, Spapen HD (2015) Intertwining extracorporeal membrane oxygenation and continuous renal replacement therapy: sense or nonsense? Crit Care 19:145

R. Jacobs · P. M. Honore (®) · H. Spapen ICU Department, Universitair Ziekenhuis Brussel, Vrije Universiteit Brussel, 101, Laarbeeklaan, Jette, 1090 Brussels, Belgium e-mail: Patrick.Honore@az.vub.ac.be Tel.: 003224749097 